\title{
Epidemiologia molecolare di isolati clinici di P. aeruginosa MDR produttori di metallo- $\beta$-lattamasi di tipo VIM-I in una struttura di neuroriabilitazione
}

\author{
Roberta Migliavacca', Antonella Navarra², Fabiola Martino', Andrea Negri², \\ Melissa Spalla ${ }^{3}$, Elisabetta Nucleo', Laura Pagani' \\ 'Università di Pavia, 'Laboratorio di Microbiologia IRCCS “Fondazione S. Maugeri”, Pavia, \\ 3Laboratorio di Microbiologia IRCCS “S. Matteo”, Pavia
}

Key words: $P$. aeruginosa, MDR, VIM-type MBL, neurorehabilitation

Molecular epidemiology of MDR P. aeruginosa VIM-I producer in a neurorehabilitation facility

\section{SUMMARY}

The VIM- and IMP-type metallo-ß-lactamases (MBLs) are clinically relevant emerging resistance determinants in Gram-negative nosocomial pathogens. To date, MBLs have been reported in acute care hospitals, but their epidemiology in Rehabilitation Facilities (RF) - the largest reservoirs of patients colonized by multidrug-resistant isolates - is largely unknown. This study is aimed to investigate MBL production in Pseudomonas aeruginosa isolates from "Fondazione S. Maugeri" Hospital an RF located in Pavia. 59 non replicate P. aeruginosa strains were isolated between July 2003 - March 2004, from patients incoming from different Italian hospitals. I8/59 resulted resistant to carbapenems. Phenotypic detection of the MBL producers was performed using the E-test and the EPI microdilution method. Molecular methods were used to investigate the MBL determinants and their genetic context. The clonal relatedness of the isolates were studied by PFGE. I2/18 carbapenem-resistant isolates were found to produce a VIM-type MBL. 7/I 2 were clonally related suggesting a clonal spread, while the 5 others, unrelated to the epidemic profile $A$, were simultaneously present in the same RF. Preventive and control measures including established microbiological protocols should be applied to detect the MBL producers and to contrast the diffusion of carbapenem-resistant $P$. aeruginosa in RF.

\section{INTRODUZIONE}

Pseudomonas aeruginosa rappresenta, nell'ambito dei batteri Gram-negativi non fermentanti, un patogeno opportunista di frequente riscontro, responsabile di infezioni gravi in pazienti debilitati che sono sottoposti ad intensa terapia antibiotica.

L'isolamento di ceppi multiresistenti (MDR) è ormai oggi molto diffuso in ambiente ospedaliero visto l'uso inappropriato ed incontrollato degli antibiotici ß-lattamici, in particolare di carbapenemici (8).

La resistenza ai $\beta$-lattamici di comune impiego può essere dovuta ad una iperproduzione della $\beta$ lattamasi AmpC cromosomica, all'acquisizione di $ß$-lattamasi secondarie e/o ad un aumentato efflusso o all'impermeabilità di membrana $(5,6$, 7,9).

Tra le $\beta$-lattamasi in grado di conferire resistenza ai carbapenemici le metallo- $\beta$-lattamasi (MBL) rappresentano gli enzimi di maggior rilievo clinico ed epidemiologico $(1,11,15)$.

Le MBL, di tipo VIM o IMP, appartengono alla classe molecolare B e sono caratterizzate dalla presenza nel loro sito attivo di ioni metallici, solitamente zinco.

Questi enzimi conferiscono resistenza a tutti i ßlattamici, inclusi i carbapenemici e gli inibitori convenzionali, mentre, non agiscono nei confronti dei monobattamici e possono essere inibiti da chelanti di ioni metallici (EDTA) (21).

Fino ad oggi, sono state descritte MBL di tipo VIM in ospedali per acuti, ma la loro epidemiologia nelle strutture di riabilitazione rimane ampiamente sconosciuta. Molti lavori segnalano, anche in Italia, la diffusione di ceppi di $P$. aeruginosa produttori di $\operatorname{MBL}(19,4,12,10)$.

D'altra parte, la gestione dei pazienti con lesioni neurologiche, sia traumatiche che non traumatiche, è migliorata considerevolmente negli ultimi anni.

Dopo la stabilizzazione, la maggior parte dei pazienti è trasferita alle unità di riabilitazione. Questi reparti costituiscono, perciò, un ampio "reservoir" di pazienti colonizzati e/o infetti da germi MDR; è importante ricordare, inoltre, che $\mathrm{i}$ 
pazienti in riabilitazione provengono da diversi ospedali italiani dove potrebbero essere già stati colonizzati da patogeni opportunisti nei reparti di provenienza.

Lo scopo dello studio è stato quello di monitorare la diffusione della resistenza ai carbapenemici, mediata da MBL, in ceppi di $P$. aeruginosa isolati in una struttura riabilitativa che accoglie pazienti provenienti da ospedali distribuiti sul territorio nazionale, caratterizzando genotipicamente i ceppi produttori e verificando una possibile correlazione epidemiologica degli isolati.

\section{MATERIALI E METODI \\ Isolati clinici.}

L'IRCCS "Fondazione S. Maugeri” di Pavia è una struttura ospedaliera che include reparti riabilitativi, di lungodegenza e che accoglie pazienti provenienti da ospedali distribuiti sul territorio nazionale.

Nel periodo Luglio 2003-Marzo 2004 sono stati isolati da diversi materiali patologici di pazienti ricoverati presso il reparto di neuroriabilitazione, 59 stipiti di P. aeruginosa non replicati, di cui 18 erano resistenti all'imipenem (IPM). Il reparto di neuroriabilitazione, con disponibilità di 43 posti letto, ha accolto nel periodo di studio 194 pazienti.

\section{Test di sensibilità in vitro}

I ceppi sono stati identificati e saggiati per l'attività antimicrobica mediante Vitek system II (bioMérieux). La MIC è stata determinata in terreno liquido con micrometodo in accordo con il protocollo del Clinical Laboratory Standard Institute (CLSI) (3). Su tutti gli isolati è stato effettuato il test di sinergia del doppio disco. Una sospensione del microrganismo in esame con torbidità $0.5 \mathrm{McF}$ è seminata su Muller-Hinton agar; i dischetti contenenti ceftazidime (CAZ), aztreonam (ATM), cefepime (FEP) vengono disposti ad una distanza di $25 \mathrm{~mm}$ attorno ad un dischetto centrale contenente amoxicillina/acido clavulanico (AMC). La presenza di caratteristiche distorsioni dell'alone di inibizione indica un'azione sinergica tra acido clavulanico, inibitore delle $\beta$ lattamasi a spettro allargato, ed antibiotico, e, quindi, la produzione di ES $\beta$ L. In caso di negatività il test viene ripetuto avvicinando i dischetti (13). I ceppi $P$. aeruginosa ATCC 27853 e $P$. aeruginosa 87SM (iperproduttore di AmpC) sono stati utilizzati come controlli negativi e $P$. aeruginosa Pa 34SM (PER-1 produttore) come controllo positivo (17).

\section{Rivelazione delle MBL}

Lo screening per la produzione di MBL è stato eseguito con E-test MBL strip (AB Biodisk, Solna, Sweden) seguendo le linee guida raccomandate dal produttore. È stato, inoltre, eseguito
l'EPI test in micropiastra con concentrazioni di IPM tra i 512 e $0.25 \mathrm{mg} / \mathrm{L}$; la MIC è stata valutata in assenza e in presenza di EDTA $(0.4 \mathrm{M})+$ 1,10-o-fenantrolina (0.04M) (14).

\section{Caratterizzazione delle $\beta$-lattamasi}

L'estratto enzimatico grezzo, ottenuto a partire da brodocolture overnight è stato sottoposto ad isoelettrofocusing (IEF). L'attività delle $\beta$-lattamasi è stata valutata, dopo IEF, nei confronti di ossimino-cefalosporine ed aztreonam, mediante la procedura del substrato ricoprente (16).

\section{Analisi molecolare}

I determinanti di resistenza sono stati individuati con PCR mediante primer specifici per i geni bla $_{\mathrm{VIM}}$ e bla Iмр. $_{\text {. }}$. aeruginosa VR-143/97 (produttore di VIM-1), $P$. aeruginosa 101/1477 (produttore di IMP-1) e $P$. aeruginosa ATCC 27853 sono stati inclusi, rispettivamente, come controlli positivi e negativo.

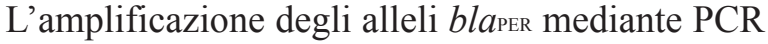
è stata eseguita utilizzando la coppia di primer BLA-PER/F (5'-GGGACARTCSKATGAATGTCA) e BLA-PER/R (5'-GG YSGCTTAGATAGTGCTGAT), come già descritto (18).

L'amplificazione degli alleli bla vім mediante PCR è stata eseguita utilizzando la coppia di primers VIM/F (5'-CAG ATT GCC GAT GGT GTT TGG-3') e VIM/R (5'-AGG TGG GCC ATT CAG CCA GA-3'), adatti per l'amplificazione della regione centrale del gene di 523 bp.

I campioni sono stati sottoposti ad una denaturazione iniziale a $94^{\circ} \mathrm{C}$ per 30 " e quindi sottoposti a 30 cicli di amplificazione con denaturazione a $94^{\circ} \mathrm{C}$ per $60^{\prime \prime}$, annealing a $55^{\circ} \mathrm{C}$ per $60^{\prime \prime}$, estensione a $72^{\circ} \mathrm{C}$ per $60^{\prime \prime}$; allungamento a $72^{\circ} \mathrm{C}$ per $10^{\prime}$. Le stesse condizioni sono state applicate per l'amplificazione degli alleli bla IMP utilizzando i seguenti primers: IMP/F (5'-GTGATGCGTCVCCAAVTTCACT-3') e IMP/R (5'-GGAATAGAGTGGCTTAATTCTC-3').

\section{Analisi dei profili di macrorestrizione genomi- ca (PFGE)}

I profili di macrorestrizione del DNA genomico sono stati ottenuti mediante la procedura Gene Path Bio-Rad (Bio-Rad Laboratories, Richmond, Calif.) utilizzando il kit di reagenti \#3 e l'enzima di restrizione SpeI.

Concatenomeri del batteriofago $\lambda$ (Bio-Rad) sono stati utilizzati per la valutazione del peso molecolare dei frammenti di restrizione visualizzati sotto luce UV dopo colorazione con bromuro d'etidio. Le relazioni clonali sono state interpretate sulla base dei criteri proposti da Tenover et al.(20).

\section{RISULTATI}

Nel periodo Luglio 2003-Marzo 2004 sono stati isolati, da pazienti ricoverati presso il reparto di 
neuroriabilitazione dell'I.R.C.C.S. "Fondazione S. Maugeri", 59 stipiti di $P$. aeruginosa non replicati, di cui 18 erano intermedi/resistenti all'IPM mediante il sistema automatizzato Vitek System II. Le MIC sono risultate comprese tra $8 \mu \mathrm{g} / \mathrm{mL}$ e $>256 \mu \mathrm{g} / \mathrm{mL}$. Di questi, 12 mostravano un profilo MDR che includeva aminoglicosidi, fluorochinoloni, ossimino-cefalosporine e piperacillina; tutti i campioni erano sensibili alla colistina, mentre 3 isolati mostravano sensibilità a piperacillina/tazobactam. I 18 ceppi IPM-resistenti sono stati isolati con maggior frequenza da urine (37\%), seguite da tamponi-ferita (21\%), secrezioni delle vie respiratorie $(21 \%)$ ed altri materiali $(21 \%)$. La produzione delle MBL è stata studiata mediante EPI test ed E-test MBL; i due test hanno fornito lo stesso risultato di positività nei 12 isolati MDR facendo sospettare così la produzione di una MBL. Inoltre 5/12 sono risultati positivi per la produzione di una ES $\beta \mathrm{L}$ con test del doppio disco. I 12 campioni mostravano la presenza di due bande $\beta$-lattamasiche con pI pari a 5.2, tipica di un enzima di tipo VIM, ed una a pI 8.0 attribuibile all'enzima AmpC-type costitutivo. Tra questi, 5 campioni, caratterizzati da multiresistenza a tutti i $\beta$-lattamici, inclusi aztreonam e piperacillina/tazobactam, mostravano una banda aggiuntiva con pI 5.3, attribuibile ad una ESBL. Mediante amplificazione genica i campioni sono risultati produrre l'enzima codificato dal gene bla a confermare il risultato dell'IEF, 5 di questi, sono risultati anche produttori di una ES $\beta \mathrm{L}$ di tipo PER-1 (figura I, II; tabella 1).

Dall'analisi di macrorestrizione è stato possibile evidenziare la presenza di 5 cloni di cui il più diffuso (clone A), presente in 7/12 isolati, ha dato origine ad un evento epidemico all'interno del reparto. Il clone B caratterizzava due isolati; i rimanenti stipiti non strettamente correlati fra loro, coesistevano nello stesso reparto di neuroriabilitazione (figura III).

Un isolato di $P$. aeruginosa VIM-1 positivo appartenente al clone $\mathrm{E}$, ottenuto da 1 paziente al ricovero, dimostra la presenza di questi geni nelle strutture ospedaliere di provenienza. L'analisi delle caratteristiche cliniche dei pazienti ha evidenziato il ruolo patogeno dei ceppi produttori di MBL.

In accordo con le definizioni di infezione ospedaliera del CDC (2), in 3 pazienti gli isolati sono stati classificati come responsabili di infezione, in 4 pazienti come colonizzatori, nei restanti 5 pazienti gli isolati sono stati classificati come possibili responsabili di infezione quando, per l'eradicazione del microrganismo, il medico ha prescritto una terapia antibiotica anche in assenza di sintomi. Tutti i pazienti erano stati sottoposti, nel periodo precedente l'isolamento, a terapia con uno o più farmaci quali ciprofloxacina, cefotaxime, ceftazidime, IPM, piperacillina-tazobactam, gentamicina, trimetropim-sulfametossazolo, vancomicina, tetraciclina, fluconazolo.

Tabella I. Caratteristiche dei 12 isolati VIM produttori

\begin{tabular}{lccccc}
\hline Ceppo Campione & $\begin{array}{c}\text { Data di } \\
\text { ammissione }\end{array}$ & $\begin{array}{c}\text { Data di } \\
\text { positività }\end{array}$ & $\begin{array}{c}\text { pl delle } \\
\text { B-lattamasi }\end{array}$ & clone \\
\hline 67 & Urocoltura & 14.07 .03 & 21.07 .03 & $8.0 ; 5.2$ & $\mathrm{~B}$ \\
\hline 76 & T. auricolare & 07.08 .03 & 28.08 .03 & $8.0 ; 7.8 ; 5.2$ & $\mathrm{C}$ \\
\hline 81 & T. ferita & 18.09 .03 & 25.09 .03 & $8.0 ; 5.2$ & $\mathrm{BI}$ \\
\hline 85 & Urocoltura & $\mathbf{I I} .09 .03$ & 02.10 .03 & $8.0 ; 5.7 ; 5.3 ; 5.2$ & $\mathrm{~A}$ \\
\hline 86 & Urocoltura & 17.09 .03 & 07.10 .03 & $8.0 ; 5.7 ; 5.3 ; 5.2$ & $\mathrm{~A}$ \\
\hline 88 & T. ferita & 18.09 .03 & 14.10 .03 & $8.0 ; 7.8 ; 5.2$ & $\mathrm{D}$ \\
\hline 90 & Urocoltura & 19.09 .03 & 16.10 .03 & $8.0 ; 5.7 ; 5.3 ; 5.2$ & $\mathrm{~A}$ \\
\hline 98 & T. decubito & 18.04 .03 & 06.11 .03 & $8.0 ; 5.2$ & $\mathrm{Al}$ \\
\hline 100 & Urocoltura & 19.10 .03 & 08.11 .03 & $8.0 ; 5.7 ; 5.3 ; 5.2$ & $\mathrm{~A}$ \\
\hline 101 & T. decubito & 08.09 .03 & 10.11 .03 & $8.0 ; 7.8 ; 5.2$ & $\mathrm{~A} 2$ \\
\hline 106 & Urocoltura & 17.11 .03 & 20.12 .03 & $8.0 ; 5.7 ; 5.3 ; 5.2$ & $\mathrm{~A}$ \\
\hline 134 & Urocoltura & 08.03 .04 & 09.03 .04 & $8.0 ; 5.2$ & $\mathrm{E}$ \\
\hline
\end{tabular}

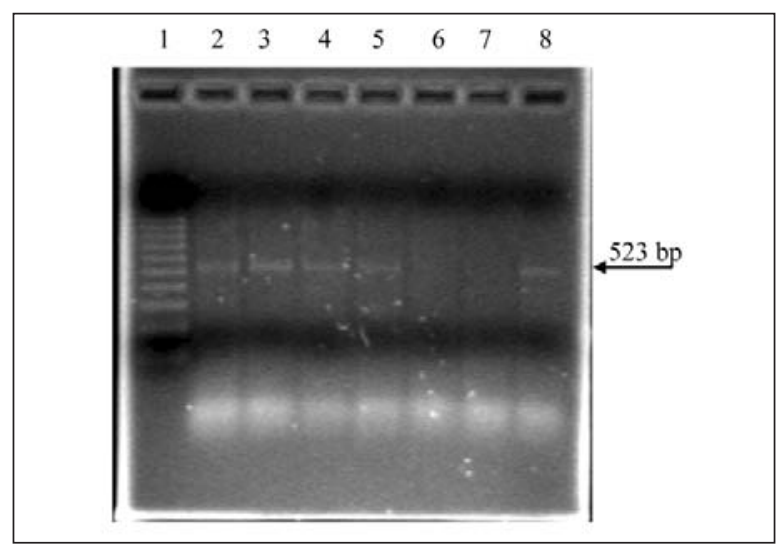

Figura I. PCR di alcuni isolati scelti come rappresentativi di P. aeruginosa produttori di metallo B-lattamasi. Linea I marker $100 \mathrm{bp}$, linea 2-5 ceppi VIM produttori; linea 6-7 isolati VIM negativi; linea 8 controllo positivo.

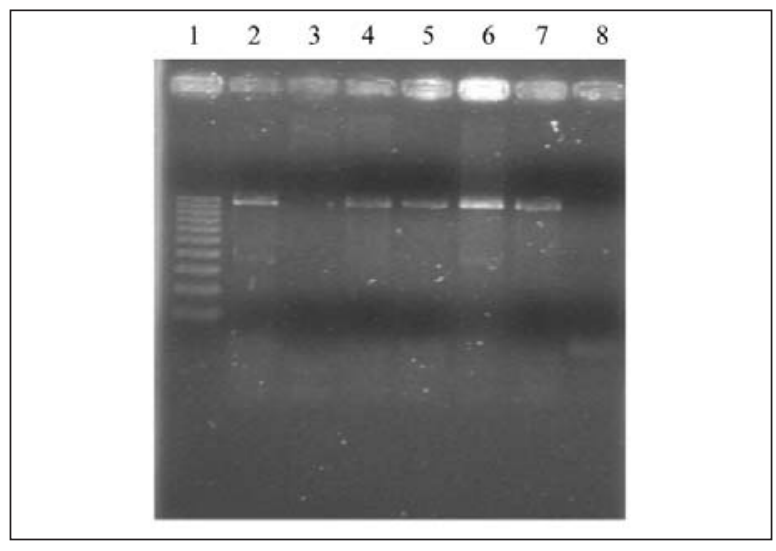

Figura II. PCR degli isolati di P. aeruginosa produttori di B-lattamasi PER-I.

Linea I marker l00bp, linea 2 controllo positivo, linea 3 isolato PER negativo, linea 4-7 ceppi PER produttori, linea 8 controllo negativo. 


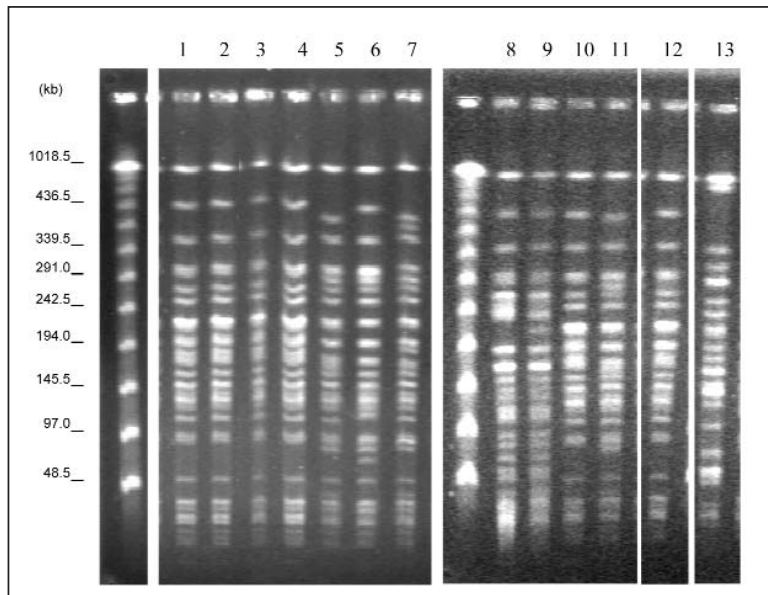

Figura III. Profilo PFGE dei 12 isolati VIM produttori. Linee: I-4, 10, 12 Pulsotipo A; linea 5: pulsotipo C; linea 6 pulsotipo $A$ I; linea 7 pulsotipo D; linea 8-9 pulsotipi $B$ e $B$ I; linea II pulsotipo A2; linea 13 pulsotipo $E$.

\section{DISCUSSIONE}

L'acquisizione di batteri MDR in una struttura riabilitativa può essere una conseguenza della pressione selettiva esercitata dall'ampio uso di antibiotici e/o dalla disseminazione orizzontale. La distinzione fra questi due meccanismi può essere fatta grazie all'uso di metodi di tipizzazione molecolare: un unico genotipo è presente nella trasmissione orizzontale, mentre molteplici cloni possono essere rilevati quando la pressione selettiva rappresenta il meccanismo più importante. L'identificazione e la tipizzazione molecolare ai fini epidemiologici dei microrganismi patogeni ospedalieri sono indispensabili per stabilire le relazioni tra gli stipiti isolati.

I risultati ottenuti dimostrano che le strutture riabilitative rappresentano un "reservoir" di $P$. aeruginosa MDR produttori di MBL di tipo VIM1 , in quanto entrambi i meccanismi di trasmissione hanno determinato la diffusione della resistenza. È evidente che la resistenza ai carbapenemici mediata dalle MBL e riscontrata non solo negli ospedali per acuti, sta assumendo dimensioni preoccupanti. Dovrebbero essere applicate, quindi, misure preventive e di controllo al fine di individuare i ceppi produttori di MBL e contrastare la diffusione di P. aeruginosa MDR. È necessario includere rigorosi protocolli microbiologici e verificare la colonizzazione dei pazienti al ricovero, dal momento che nella struttura riabilitativa afferiscono pazienti da ospedali diversi per dimensione e per tipologia.

L'utilizzo di test di sinergia con inibitori per il riconoscimento delle MBL è consigliabile per i laboratori diagnostici ed è di facile applicazione al fine di monitorare la persistenza e la diffusione di diversi cloni MBL produttori. Infine, la pronta notifica di microrganismi "sentinella" con quadri di resistenza inusuale, contribuisce a migliorare le misure di controllo ed a diminuire l'instaurarsi di epidemie nosocomiali.

\section{BIBLIOGRAFIA}

1. Bush K. New B-lactamases in Gram-negative bacteria: diversity and impact on the selection of antimicrobial therapy. Clin Infect Dis 2001; 32: 1085-9.

2. CDC definitions for nosocomial infections. In: Olmsted RN, ed.: APIC Infection Control and Applied Epidemiology: Principles and Pratice. St. Louis: Mosby 1996; A 1-20.

3. Clinical and Laboratory Standards Institute. Performance standards for antimicrobial susceptibility testing; fifteenth informational supplement. M100S15. Clinical and Laboratory Standards Institute. Wayne, $\mathrm{Pa} 2005$.

4. Cornaglia G, Mazzariol A, Lauretti L, Rossolini GM, Fontana R. Hospital outbreak of carbapenem-resistant Pseudomonas aeruginosa producing VIM-1, a novel transferable metallo-beta-lactamase. Clin Infect Dis 2000; 31(5): 1119-25

5. De Freitas AL, Barth AL. Antibiotic resistance and molecular typing of Pseudomonas aeruginosa: focus on imipenem. Braz J Infect Dis 2002; 6: 1-7

6. Higgins PG, Fluit AC, Milatovic D, Verhoef J, Schmitz FJ. Antimicrobial susceptibility of imipenem-resistant Pseudomonas aeruginosa. J Antimicrob Chemoter 2002; 50: 299-301

7. Howard DH, Scott II RD, Packard R, Jones D. The global impact of drug resistance. Clin Infect Dis 2003 36: S4-S10.

8. Jawets, Melnick, Adelberg's. Pseudomonas, Acinetobacter e batteri Gram-negativi rari. In: Microbiologia medica. Piccin editore $21^{\circ}$ edizione. Padova 2001; 241-5.

9. Jean SS, Teng LJ, Hsueh PR, Ho SW, Luh KT. Antimicrobial susceptibilities among clinical isolates of extended spectrum cephalosporin-resistant Gramnegative bacteria in a Taiwanese university hospital. Antimicrob Agents chemoter 2002; 49: 69-76.

10. Lauretti L, Riccio ML, Mazzariol A, Cornaglia G, Amicosante G, Fontana R, Rossolini GM. Cloning and characterization of bla $\mathrm{VIM}$, a novel integronborne metallo- $\beta$-lactamase gene from a Pseudomonas aeruginosa clinical isolate. Antimicrob Agents Chemoter 1999; 43: 1584-90.

11. Livermore DM, Woodford N. Carbapenemases: a problem in waiting? Curr Opin Microbial 2000; 3: 48995.

12. Lombardi G, Luzzaro F, Docquier JD, et al. Nosocomial infections caused by multidrug-resistant isolates of Pseudomonas putida producing VIM-1 metallo-beta-lactamase. J Clin Microbiol 2002; 40(11): 4051-5.

13. Luzzaro F, Mantengoli E, Perilli M, et al Dynamics of a nosocomial outbreak of multidrug-resistant Pseudomonas aeruginosa producing the PER-1 extended-spectrum $\beta$-lactamase. J Clin Microbiol 2001; 39: 1865-70.

14. Migliavacca R, Docquier JD, Mugnaioli C, et al. Simple microdilution test for detection of metallobeta-lactamase production in Pseudomonas aeruginosa. J Clin Microbiol 2002; 40(11): 4388-90. 
15. Nordmann P, Poirel L. Emerging carbapenemases in Gram-negative aerobes. Clin Microbiol Infect 2002; 8: 321-31.

16. Pagani L, Ronza P, Giacobone E, Romero E. Extended-spectrum beta-lactamases from Klebsiella pneumoniae strains isolated at an Italian hospital. Eur J Epidemiol 1994; 10(5): 533-40.

17. Pagani L, Mantengoli E, Migliavacca R, et al. Multifocal detection of multidrug-resistant Pseudomonas aeruginosa producing the PER-1 extended-spectrum beta-lactamase in Northern Italy. J Clin Microbiol 2004; 42(6): 2523-9.

18. Pereira M, Perilli M, Mantengoli E, Luzzaro F, Toniolo A, Rossolini GM, Amicosante G. PER-1 extended-spectrum $\beta$-lactamase production in an Alcaligenes faecalis clinical isolate resistant to expandedspectrum cephalosporins and monobactams from a hospital in Northern Italy. Microb Drug Resist 2000; 6: 85-90.

19. Rossolini GM, Riccio ML, Cornaglia G, et al. Carbapenem-resistant Pseudomonas aeruginosa with acquired bla(vim) metallo-beta-lactamase determinants, Italy. Emerg Infect Dis 2000; 6(3): 312-3.

20. Tenover FC, Arbeit RD, Goering RV, Mickelsen PA, Murray BE, Persing DH, Swaminathan B. Interpreting chromosomal DNA restriction patterns produced by pulsed-field gel electrophoresis: criteria for bacterial strain typing. J Clin Microbiol 1995; 33: 2233-9.

21. Walsh TR, Toleman MA, Poirel L, Nordman P. Metallo-ß-Lactamases: the quiet before the storm? Clin Microbiol Rev 2005; 18(2): 306-25.

\section{Laura Pagani}

Via Brambilla 74, 27100 Pavia

Tel. 03825484206

Fax 03825484255

E-mail: Ipagani@unipv.it 